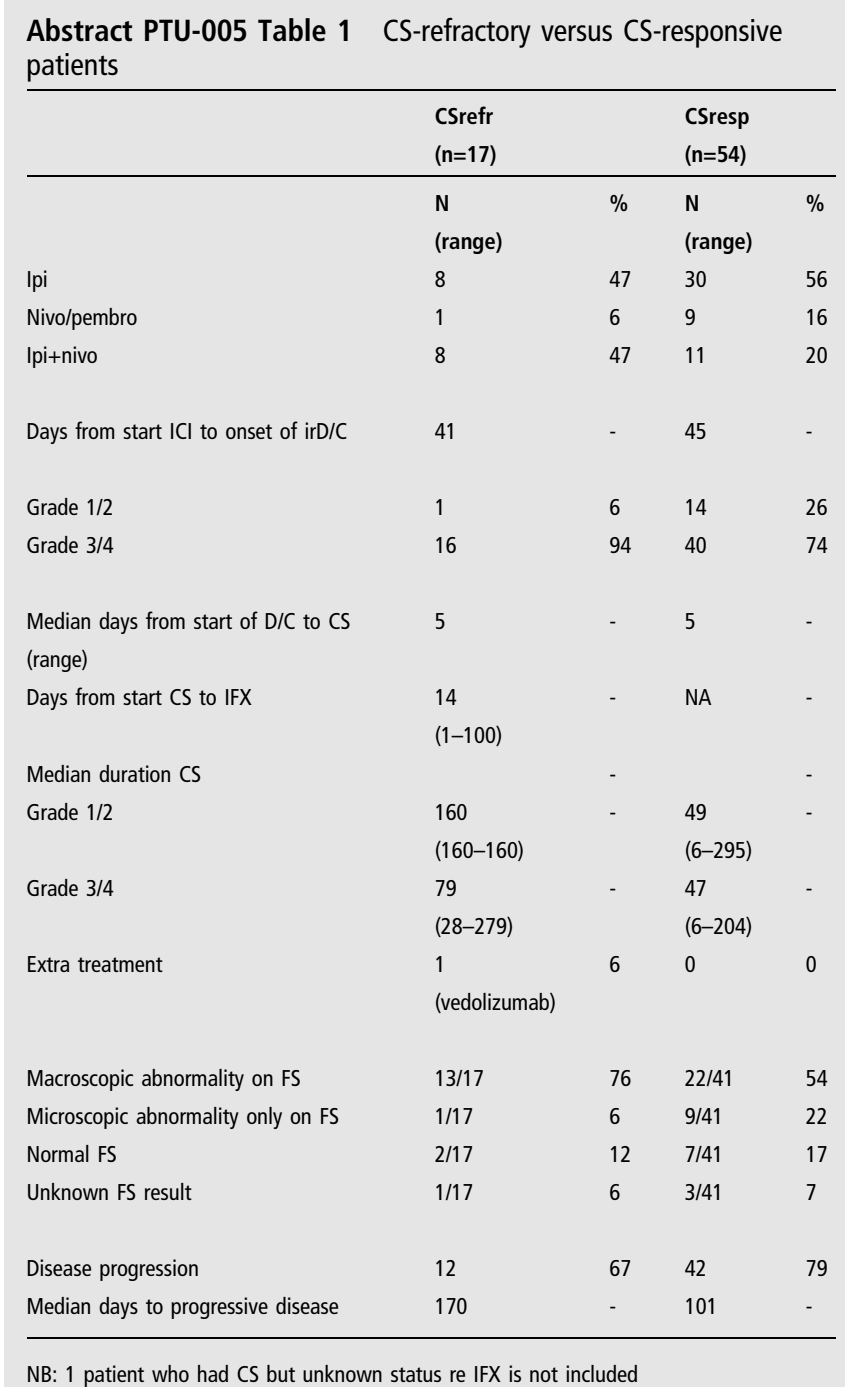

\section{PTU-006 INCIDENCE OF IMMUNE CHECK POINT INHIBITOR INDUCED DIARRHOEA/COLITIS- EXPERIENCE FROM A MULTI-CENTRE COHORT}

${ }^{1}$ Lavinia Spain*, ${ }^{2}$ Amit Samani, ${ }^{1,2}$ Hajir Ibraheim, 'Lewis Au, ${ }^{2}$ Zayd Tippu, ${ }^{2}$ Shuai Zhang, ${ }^{2}$ Sophie Merrick, ${ }^{2}$ Debra Josephs, ${ }^{2}$ Waqas Fazal, ${ }^{2}$ Sophie Papa, ${ }^{1,2}$ Nick Powell, ${ }^{1}$ Nadia Yousaf, ${ }^{1} J a m e s$ Larkin, ${ }^{1}$ Martin Gore, ${ }^{1}$ Samra Turajic. ${ }^{1}$ Royal Marsden NHS Foundation Trust, London, UK; ${ }^{2}$ Guys and St Thomas' NHS Foundation Trust, London, UK

\subsection{6/gutjnl-2018-BSGAbstracts. 128}

Background Immune checkpoint inhibitors (ICIs) including anti-CLTA-4 (e.g. ipilimumab (ipi)) and anti-PD-1 antibodies (e.g. nivolumab (nivo)) have improved outcomes in many cancers. However their use is complicated by ICI-related diarrhoea/colitis (irD/C), a common cause of morbidity and ICI discontinuation. The National Cancer Institute's Common Terminology Criteria for Adverse Events (CTCAE) has been used to grade $\mathrm{irD} / \mathrm{C}$ according to frequency of bowel movements over baseline. Grade 1-2 represents mild-moderate disease, grade 3-4 severe disease and grade 5 represents death. In clinical trials diarrhoea/colitis is more common in regimes using anti-CTLA-4 agents. ${ }^{1}$ There are few real world data reported in the UK.
Methods Electronic medical records were reviewed for melanoma patients (pts) at The Royal Marsden Hospital (RMH) and melanoma, renal and lung cancer pts Guy's and St Thomas' Hospital (GSTT), receiving at least one ICI dose between 2011-2016. Clinical outcome data included class of ICI therapy and CTCAE grade of diarrhoea.

Results 651 ICI treatment courses were administered mostly for melanoma (100\% RMH, 53\% GSTT). 285 (44\%) received anti-CTLA-4 monotherapy, 288 (44\%) anti-PD-1 monotherapy, and $77(12 \%)$ combination ipi + nivo. The incidence of allgrade $\mathrm{irD} / \mathrm{C}$ was $27 \%$ for anti-CTLA-4 therapy, $12 \%$ for antiPD-1\%-34\% for ipi + nivo. The incidence of severe $\mathrm{irD} / \mathrm{C}$ (grade $3-5$ ) was $12 \%$ in anti-CTLA-4 monotherapy, $4 \%$ in anti-PD-1 therapy and $26 \%$ in combination therapy (figure 1). There was one only death reported in a pt who developed colitis following treatment with anti-CTLA-4 monotherapy.

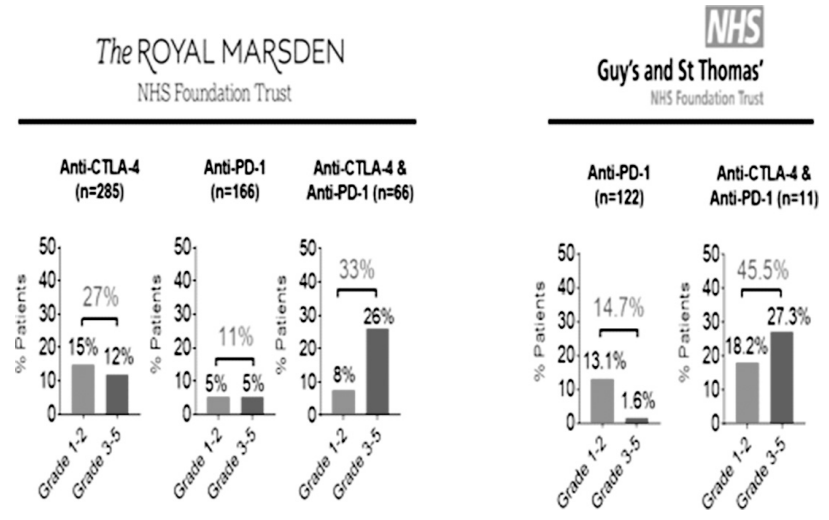

Abstract PTU-006 Figure 1

Conclusion This is the largest cohort of data reporting the incidence of $\mathrm{irD} / \mathrm{C}$ involving real-world patients. Compared to trial data, the incidence of all-grade diarrhoea was slightly lower but the incidence of severe disease was higher in all treatment groups, particularly with ipi + nivo. Given the expansion of ICIs in other cancer types and use as an adjuvant therapy, there is an urgent need to engage gastroenterology services and to develop evidence-based management algorithms for treatment of $\mathrm{irD} / \mathrm{C}$.

\section{REFERENCE}

1. Spain L, Diem S, Larkin J. Management of toxicities of immune checkpoint inhibitors. Cancer Treat Rev 2016;44:51-60.

\section{PTU-007 A RETROSPECTIVE MULTICENTRE STUDY COMPARING INFLIXIMAB AND ADALIMUMAB FOR MAINTENANCE OF REMISSION IN ULCERATIVE COLITIS}

${ }^{1}$ Victoria Kronsten*, ${ }^{1}$ Michael Colwill, ${ }^{2}$ Shadab Nayeemuddin, ${ }^{2}$ Jimmy Limdi, ${ }^{3}$ Christian Selinger, ${ }^{4}$ Glyn Scott, ${ }^{5}$ Lulia Al-Hillawi, ${ }^{5}$ Shayon Salehi, ${ }^{5}$ Paul Blaker, ${ }^{1}$ Guy ChungFaye, ${ }^{1}$ Alexandra Kent, ${ }^{1}$ Patrick Dubois, ${ }^{1}$ Bu Hayee. ${ }^{1}$ King's College Hospital NHS Trust, London, U.K. ${ }^{2}$ The Pennine Acute Hospitals NHS Foundation Trust, Manchester, U.K. ${ }^{3}$ Leeds Teaching Hospitals NHS Trust, Leeds, U.K; ${ }^{4}$ East Kent Hospital University Foundation Trust, Margate, U.K; ${ }^{5}$ Maidstone and Tunbridge Wells NHS Trust, Tunbridge Wells, U.K

\subsection{6/gutjnl-2018-BSGAbstracts. 129}

Introduction Anti-tumour necrosis factor (TNF) therapy has revolutionised ulcerative colitis (UC) treatment, particularly in 
moderate-to-severe disease. However, these drugs tend to perform less well in the maintenance of remission. Route of administration may influence efficacy and network meta-analyses of trial data indicate a superiority of intravenous drugs (IV; Infliximab; IFX) over subcutaneous (SC; adalimumab; ADA). We conducted a retrospective multicentre case-control study to compare the efficacy of these two drugs.

Methods Patients administered IFX or ADA as their first biologic, identified from therapy databases of five UK hospitals, were included, if they had completed induction dosing and entered maintenance. Patients receiving IFX as 'rescue' therapy were excluded. Data was collected for pre-biologic disease activity (Simple Clinical Colitis Activity Index (SCCAI), Creactive protein and calprotectin) and throughout anti-TNF therapy. The primary end-point for comparison was the number of patients in clinical remission at 52 weeks (combined features of continuing IFX or ADA therapy and SCCAI score $\leq 3$ ). Data was collected for duration of therapy, or up to last follow-up, if beyond 52 weeks.

Results 78 IFX $(40.3 \pm 14.6$ years, 33F) and 63 ADA (36.8 \pm 14.6 years, $27 \mathrm{~F}$ ) patients were analysed. There were no statistically significant differences in demographics or pre-biologic disease activity between the two groups. At 52 weeks, 58 (74\%) IFX patients and 29 (46\%) ADA patients remained on therapy $(\mathrm{p}=0.009)$ and in remission $(26(33 \%)$ vs $5(8 \%)$, $\mathrm{p}=0.0003)$. Primary non response was the reason for treatment cessation in 15 (24\%) ADA patients and 4 (5\%) IFX patients $(\mathrm{p}=0.0012)$.

Conclusions Our results from a real-world cohort mirror those produced in the network meta-analyses of clinical trials for these agents, suggesting that IFX is superior to ADA in UC maintenance of remission, demonstrated by improvement in SCCAI scores and treatment continuation at 52 weeks. There were no significant differences in colectomy rates, hospital admission for acute flares or adverse events in the study timeframe.

\section{REFERENCE}

1. Danese S, Fiorino G, Peyrin-Biroulet L, Lucenteforte E, Virgili G, Moja L, Bonovas S, (2014), Biological agents for moderately to severely active ulcerative colitis: a systematic review and network meta-analysis., Ann Intern Med, 160:704-11.

\section{PTU-008 INTERLEUKIN 23 AS A NON-INVASIVE TEST OF DISEASE SEVERITY IN PATIENTS WITH ULCERATIVE COLITIS}

\footnotetext{
${ }^{1,2}$ Angie Rund*, ${ }^{2}$ Tarek Youssef, ${ }^{2}$ Shereen Saleh, ${ }^{2}$ Iman Fawzy, ${ }^{2}$ Manal Mohsen, ${ }^{2}$ Ola Hazem. ${ }^{1}$ Royal Liverpool University Hospital, Liverpool, UK; ${ }^{2}$ Ain Shams University Hospitals, Cairo, Egypt
}

\subsection{6/gutjnl-2018-BSGAbstracts. 130}

Introduction Studies have found increased expression of IL-23 in inflamed and non-inflamed mucosa of patients with ulcerative colitis (UC). This study was done to evaluate serum interleukin-23 as a non-invasive test for ulcerative colitis disease and assessed its correlation with the disease severity.

Methods A prospective case-control study where 80 patients were recruited, and allocated into two groups:

Group I: included forty patients diagnosed with UC by clinical, endoscopic and histopathologic features.

Group II (control group): included 40 patients without UC, matched in age and gender, who had colonoscopy for various indications but had a normal colonoscopy and normal histopathology.

In patients with UC, disease severity was assessed using the Mayo Scoring System for assessing UC activity. Serum IL-23 level was quantified using Quantikine Human IL-23 Immunoassay by $\mathrm{R}$ and D Systems Europe, Ltd. ELISA kit. IL-23 levels were compared in the 2 groups, also correlation with severity was obtained.

Analysis of the data was done using SPSS (Statistical System for Social Science version 16). Kruskal Wallis test was used to compare the 2 groups regarding quantitative nonparametric variables. Spearman correlation was used to rank variables positively or inversely. Receiver operating curve (ROC) was used to find the best cut off and validity of IL-23. The oneway ANOVA test was used to assess the relationship between the severity of UC and IL-23 levels.

Results Patients with UC had higher level of interleukin 23 $(234.5+161 \mathrm{pg} / \mathrm{mL})$ compared to controls $(54.2+15 \mathrm{pg} / \mathrm{mL})$. A positive correlation was found between the level of IL-23 and disease severity. A cut off value of IL-23 $=68 \mathrm{pg} / \mathrm{mL}$ was the best to differentiate between cases and controls. Performing the receiver operating characteristic curve (ROC) revealed that the best cut off values of IL-23 to identify the severity of ulcerative colitis were $105 \mathrm{pg} / \mathrm{mL}$ for mild cases $(80 \%$ sensitivity), $200 \mathrm{pg} / \mathrm{mL}$ for moderate cases $(60 \%$ sensitivity), and $270 \mathrm{pg} / \mathrm{mL}$ for severe cases ( $81 \%$ sensitivity).

Conclusion Our findings reinforce the suggestion that IL-23 level measurement may be of value as a non-invasive test in the diagnosis and disease severity assessment in patients with UC. Further studies on a larger scale would be needed to evaluate whether this could be used for monitoring of response to treatment. In view of IL-23 antagonists currently being studied in UC patients, the predictability of response to IL-23 antagonists guided by IL-23 levels is an area that could be explored.

\section{PTU-009 UPPER GASTROINTESTINAL INFLAMMATION IN PATIENTS WITH IMMUNE-CHECKPOINT INHIBITOR INDUCED DIARRHOEA}

${ }^{1}$ Muhammad Waqas Fazal ${ }^{*},{ }^{2}$ Lavinia Spain, ${ }^{1}$ Hajir Ibraheim, ${ }^{3}$ Nadia Yousaf, ${ }^{2}$ Martin Gore, ${ }^{2}$ James Larkin, ${ }^{2}$ Samra Turajlic, ${ }^{1}$ Nick Powell, ${ }^{2}$ Lewis Au. ${ }^{1}$ Gastrointestinal Unit, Royal Marsden Hospital, Chelsea, UK; ' ${ }^{2}$ Kkin Unit, Royal Marsden Hospital, Chelsea, UK; ${ }^{3}$ Lung Unit, Royal Marsden Hospital, Chelsea, UK

\subsection{6/gutjnl-2018-BSGAbstracts.131}

Introduction Immune Checkpoint inhibitors (ICPi) have revolutionised the management of melanoma, non-small cell lung cancer and renal cancer. They block receptors expressed by immune cells that reduce immune activation. 'Turbo-charged' immune cells deliver augmented anti-tumour immunity (hence the striking efficacy of these anti-cancer agents), but comes at the cost of immune mediated side effects. Immune-mediated damage to the gut is a common and serious side effect of ICPi therapy. Endoscopic and histological findings in the lower gastrointestinal (GI) tract have been described (colitis is a common feature), but little is known about manifestations in the upper GI tract.

Methods We performed a retrospective analysis of all patients presenting with diarrhoea following treatment with ICPis (ipilimumab, nivolumab, pembrolizumab or combination therapy) who had been investigated with OGD. Endoscopic and 